\title{
Alfabetización Científica: Herramienta Indispensable en la Era Digital
}

\author{
Scientific Literacy: Indispensable Tool in the Digital Age
}

Ruth M. Mujica-Sequera

Recibido: 30/octubre/2020

Aceptado: 30/enero/2021

Publicado: 16/abril/2021

我País

${ }^{1}$ Omán

\section{IIIII Institución}

${ }^{1}$ Grupo Docentes 2.0 C.A

\section{Correo Eletrónico}

${ }^{1}$ ruth.mujica@docentes20.com

\section{iD ORCID}

${ }^{1}$ https://orcid.org/0000-0002-2602-5199

\section{Citar así: 4 APA / IEEE}

Mujica-Sequera, R. (2021). Alfabetización Científica: Herramienta Indispensable en la Era Digital. Revista TecnológicaEducativa Docentes 2.0, 11(1), 19-26. https://doi.org/10.37843/rted.v11i1.181

R. Mujica-Sequera, "Alfabetización Científica: Herramienta Indispensable en la Era Digital", RTED, vol. 11, n. ${ }^{\circ} 1$, pp. 1926, abr. 2021.

\section{Resumen}

Al reflexionar sobre el término alfabetización, los individuos de inmediato se imaginan a un sujeto sin competencias mínimas de lectoescritura. El presente artículo se concibió con el fin de llevar a cabo un estudio sobre la importancia de generar conocimiento en el siglo XXI. El cual, surgió del antagonismo, de que las publicaciones científicas solo deben ser publicadas por expertos. Para el emprendimiento del estudio, se sostuvo una aproximación multidisciplinar, donde se incorporaron diferentes perspectivas, así como, concepciones sobre las Ciencia Sociales, psicología, participación ciudadana, social y pedagógica. Se realizó una investigación cualitativa de enfoque fenomenológico-hermenéutico, donde se entrevistaron a diez (10) actores sociales del Taller Internacional de Redacción de Artículos Científicos, para luego estructurar, codificar hasta categorizar los resultados. Este recorrido epistémico permitió un acercamiento teórico, centrado en la alfabetización de los actores sociales, en calidad de, generar conocimiento a través de educación científica significativa. Partiendo de la voz de los actores sociales, se estableció el desgarramiento de las cadenas burocráticas de cada editorial, debido a que el conocimiento es más accesible en la era digital. No obstante, el analfabetismo se ha propagado vertiginosamente, incitada por los grupos de la vieja escuela, creando confusión en el qué de los sucesos, para expandir su hegemonía. Por ende, alfabetización científica (AC) no solo está centrada en el factor común de la actividad científica del cómo, sino también en el fortalecimiento de la ciencia a través de las Instituciones de Educación Superior, grupos de investigación, organizaciones y empresas educativas.

Palabras clave: Siglo XXI, educación, científico, herramienta, alfabetización.

\section{Abstract}

When reflecting on the term literacy, individuals immediately imagine a subject without minimum literacy skills. This article was conceived to study the importance of generating knowledge in the 21 st century. It arose from the antagonism that experts should only publish scientific publications. For the study's undertaking, a multidisciplinary approach was maintained, where different perspectives were incorporated and conceptions about Social Sciences, psychology, citizen, social and pedagogical participation. Qualitative research of the phenomenological-hermeneutic method was carried out. Ten (10) social actors of the International Workshop of Writing Scientific Articles were interviewed to structure, codify, and categorize the results. This epistemic journey allowed a theoretical approach focused on the literacy of social actors to generate knowledge through meaningful science education. Based on the social actors' voice, it was established the tearing of each editorial's bureaucratic chains because the experience is more accessible in the digital era. However, illiteracy has spread rapidly, incited by the old school groups, creating confusion in the events, and expanding their hegemony. Therefore, scientific literacy (SLC) is not only focused on the common factor of scientific activity of how, but also the strengthening of science through Higher Education Institutions, research groups, organizations, and educational enterprises.

Keywords: Legislation, constitution, law, violation of human rights, justice. 


\section{Introducción}

Desde el siglo XX, los expertos en el ámbito científico han solicitado a las instituciones educativas una alfabetización científica más significativa, que les permita a los estudiantes justificar los sucesos diarios presentes en la sociedad actual. No obstante, cuando se reflexiona sobre el término alfabetización, de inmediato los individuos imaginan a un sujeto sin competencias mínimas de lectoescritura, en otras palabras, no cuenta con competencias léxicas del idioma materno o extrajeras. Por las razones antes expuestas, se consolidan las bases de alfabetización científica (AC) sustentadas por los autores (McConney, Oliver, Woods- McConney, Schibeci \& Maor (2014) porque todavía se mantiene imperante y con nuevos retos en la era digital, lo cual, debe cumplirse bajo una competencia formativa del ciudadano actual.

Por consiguiente, el presente artículo surge del antagonismo de que las publicaciones científicas solo deben ser publicado por expertos, en otras palabras, paradójicamente, aquel que domina las competencias de escritura científica, es quién puede generar conocimiento. En tal sentido, se sostuvo una aproximación multidisciplinar, en donde se incorporan diferentes perspectivas, así como, concepciones de las ciencia sociales, psicología, participación ciudadana, social, así como pedagógica, resultando pertinente plantear una propuesta donde las instituciones educativas fomenten la adquisición de destrezas científicas ligadas a evaluación y conservación de los referentes en la era digital, tal como lo señala Olmedo-Estrada (2011) cada vez se hace más evidente que la (AC) "resulta indispensable para el ciudadano del siglo XXI" (p.145).

La AC en calidad de competencia curricular está comprometida con el avance del alfabetismo, adquisición de conocimientos, dominio de un lenguaje complejo, epistemología científica capaz de entender los límites (Aguilar,1999) con el fin de fortalecer el progreso social, económico (Hurd, 1998), percepción eficaz de la naturaleza científica (Abd- el-Khalick, Bell \& Lederman, 1998), participación activa responsablemente sobre los problemas del mundo (Martín-Díaz, 2001), asimismo, transformación curricular de las instituciones educativas que orientan la enseñanza científica hacia aspectos sociales y personales del estudiante (Furió, Vilches, Guisasola \& Romo, 2001).

El propósito de esta investigación emana de la penuria de promover la alfabetización científica desde las Instituciones de Educación Superior, grupos de investigación, organizaciones y empresas educativas porque de acuerdo con Sternberg \& Lubart (1997) la creatividad e innovación son menospreciadas por la colectividad e instituciones que existen dentro de la sociedad. Visto de este modo resulta ineludible reflexionar lo planteado por Laugksch (2000) el conocimiento científico es utilizado para participar en la toma de decisiones sobre el mundo. De allí, se puedo evidenciar los siguientes cuestionamientos: ¿Quién puede generar conocimientos científicos? ¿cómo promover la alfabetización científica? ¿cuáles son los aportes AC en la construcción del conocimiento en el siglo XXI? ¿cómo contribuye el AC en la producción científica de calidad?

\section{Material y Métodos}

Para estudiar las controversias de los diferentes autores y expertos en escritos científicos la presente investigación se sustentó en el paradigma cualitativo, bajo el enfoque fenomenológico que permitió a la autora conducirse por los senderos hermenéuticos a través de los eventos propios en la comprensión del sujeto investigado. El enfoque fenomenológico de investigación de acuerdo con el autor Husserl (1998) ambiciona revelar la naturaleza de los objetos, atributo y autenticidad de los fenómenos desde la experiencia vivida.

Al respecto, los autores Aguirre \& Jaramillo (2012) sostienen que fenomenología es método filosófico, con énfasis en los fenómenos (Heidegger, 2006). En otras palabras, en el sentido, significado y formación subjetivad el mundo (Bolio, 2012), exhibe en cada momento la experiencia humana (Rizo-Patrón, 2015). Por ello, se hizo necesario comprender desde las voces de los autores una dinámica combinada, una totalidad organizada y sistémica, empleada bajo la metodología cualitativo-estructural tal como lo señaló Martínez (1996). 
Considerando que, la fenomenología y enseñanza se constituyen a partir de la transferencia de cultura con el mundo, desde la experiencia al comprender y hablar de la realidad (San Martín, 1986, p. 9).

La hermenéutica ofreció congruencias para instaurar pericias que admiten el acercamiento del contexto organizacional, para comprender las actuaciones de los autores en el mismo. Esta investigación tuvo como contexto de desarrollo en la plataforma tecnológica - educativa Docente 2.0, la cual congrega las condiciones propicias para el abordaje del estudio. Se estableció un nuevo conocimiento a partir de la interacción social y colaboración activa por medio de la técnica de entrevista en profundidad, dirigida a diez (10) actores sociales pertenecientes al Taller Internacional de Redacción de Artículos Científicos, para Revistas Indexadas. El proceso de interpretación se realizó mediante la estructuración, codificación y categorización del contenido.

En la presente investigación, la autora no ambicionó verificar una hipótesis, sino develar lo subyacente a partir de la voz de los autores sociales; la exégesis derivada en este estudio buscó comprender, estructurar, sistematizar y codificar los testimonios, a partir de las categorías emergentes identificadas para cada unidad de significados. Para estos efectos, emergieron como unidades de significados: (1) Escritura científica, (2) currículo, metodología de la investigación y (4) cultura informacional. De éstas surgieron las subcategorías emergentes: Competencias léxicas, comunicación, experiencia, preparación profesional, conocimiento significativo, estrategias de redacción, resultados de investigación, métodos, conocimiento científico, creación de contenidos, habilidad de escritura, recursos didácticos, para un total de doce (12) categorías emergentes.

Esta metodología cumplió con el propósito de la investigación en cuanto a conocer cómo piensan y actúan los autores de producción científica desde sus prácticas de escritura, constituidas con base en la reflexión crítica compartida, para ser considerada objeto de transformación científica, por medio del pragmatismo del léxico científico que implica la resolución de problemas prácticos de escritura científica, entre ellos: artículos, tesis, ensayos y documentos reflexivos de racionalidad dinámica, desde las subjetividades individuales del autor acordes con la sociedad del conocimiento, el cual, conlleva a valorar el conocimiento empírico que construyen los autores para el avance de la ciencia.

\section{Resultados}

La relatoría hermenéutica se diseñó desde un doble enfoque, el científico-académico sobre las unidades de significados y categorías emergentes surgidas de la interacción con los actores sociales, lo cual permitió develar, algunos hallazgos sustanciales sobre el objeto de estudio, ver Tabla 1.

\section{Tabla 1}

Relación de la Unidad de Significados y Categorías Emergentes

\begin{tabular}{|c|c|c|c|}
\hline $\begin{array}{l}\text { Unidades de } \\
\text { significado }\end{array}$ & $\begin{array}{c}\text { Categorías } \\
\text { Emergentes }\end{array}$ & Codificación & Cromatización \\
\hline \multirow{4}{*}{$\begin{array}{l}\text { Escritura } \\
\text { Científica }\end{array}$} & Competencias & ECCL & Azul oscuro \\
\hline & Léxicas & & \\
\hline & Comunicación & ECC & Púrpura \\
\hline & Experiencia & ECE & Verde grama \\
\hline \multirow[t]{5}{*}{ Currículo } & Preparación & СРP & Morado claro \\
\hline & Profesional & & \\
\hline & Conocimiento & $\mathrm{CCS}$ & Rojo oscuro \\
\hline & Significativo & & \\
\hline & $\begin{array}{l}\text { Estrategias de } \\
\text { Redacción }\end{array}$ & CER & Anaranjado \\
\hline \multirow{4}{*}{$\begin{array}{l}\text { Metodología } \\
\text { de la } \\
\text { Investigación }\end{array}$} & Resultados de & MIRI & Fucsia \\
\hline & Investigación & & \\
\hline & Métodos & MMIM & Amarillo \\
\hline & $\begin{array}{l}\text { Conocimiento } \\
\text { Científico }\end{array}$ & MICC & Gris claro \\
\hline \multirow{4}{*}{$\begin{array}{l}\text { Cultura } \\
\text { Informacional }\end{array}$} & Creación de & CICC & Rojo \\
\hline & Contenidos & & \\
\hline & $\begin{array}{l}\text { Habilidad en } \\
\text { la Escritura }\end{array}$ & CIHE & $\begin{array}{c}\text { Verde } \\
\text { aceituno }\end{array}$ \\
\hline & $\begin{array}{l}\text { Recursos } \\
\text { Didácticos }\end{array}$ & CIRD & Azul claro \\
\hline
\end{tabular}

Nota. El abordaje sistemático de las relaciones categoriales permitió interpretar desde la voz de actores objeto de estudio, elaboración propia (2020).

Esta investigación permitió evaluar la importancia de generar conocimiento en el siglo XXI. En consecuencia, se determinó que es positivo alfabetizar para la construcción de contenido científico de calidad, al momento de llevar a cabo los cambios acordes con la realidad y estrategias de redacción científica en la población de objeto de estudio. De este abordaje 
ordenado, se proporcionó la interpretativa que continuación se presenta en la Tabla 2:

Tabla 2

Categoría: Escritura Científica

\begin{tabular}{ccc}
\hline \multicolumn{4}{c}{ Matriz I: Análisis e Interpretación de los resultados } \\
\hline $\begin{array}{c}\text { Unidad de } \\
\text { Significados }\end{array}$ & $\begin{array}{c}\text { Categorías } \\
\text { Emergentes }\end{array}$ & Interpretación del Autor \\
\hline & & Al interpretar el sentir de lo
\end{tabular}

$\begin{array}{cc}\text { Escritura } & \text { Competencias } \\ \text { Científica } & \text { Léxicas }\end{array}$

Al interpretar el sentir de lo expuesto por todos los actores sociales, de acuerdo con lo encontrado en sus discursos sobre la Competencias Léxicas, se pudo comprender que existe consenso en afirmar, conforme a lo expuesto por el A1, A3, A5, A7, A9 y A10, que la generación de conocimiento científico amerita alfabetización para enriquecer su experiencia al redactar con calidad, porque esto incide en mayor o menor grado en la formación de contenido de calidad en la era digital. Por otra parte, se logró afirmar que detrás de cualquier práctica educativa cotidiana siempre hay una respuesta a "por qué" y "para qué" se enseña. En los recintos académicos, la mayoría de los docentes, investigadores y facilitadores, no cuentan con experiencia en redacción de artículos científicos, en otras palabras, no cuentan con competencia léxicas en redacción científica. Por ello, el mundo en sí mismo se convierte en un escenario propicio para redefinir la experiencia pedagógica, mediante el desarrollo de capacidades para observar, reflexionar, idear estrategias y solucionar problemas prácticos. En síntesis, cavilar sobre la experiencia crea condiciones necesarias para mejorar el ámbito científico.

Nota. La competencia léxica, es aquel conocimiento y capacidad del vocabulario de una lengua para utilizarlo, elaboración propia (2020).

De lo reseñado estructura conceptual "Competencias Léxicas", se puede deducir que implica integrar, conservar y preservar la producción intelectual significativa. El autor Alfonso-Sánchez (2001) sustenta "los manuscritos y de cada una de sus ediciones revela las numerosas correcciones que experimentan las obras antes de ir, definitivamente, a la imprenta" (p.1). Por ello, las instituciones educativas, organizaciones $\mathrm{y}$ empresas dedicadas a la generación de contenido de calidad no deben olvidar la importancia del proceso de investigación, originalidad, metodología, así como, estructuras adecuadas, con la finalidad de que su publicación y difusión cumpla con los tres principios esenciales: rigor, transparencia, tal como brevedad, ver tabla 3.

\section{Tabla 3}

\section{Categoría: Currículo}

\section{Matriz I: Análisis e Interpretación de los resultados}

Unidad de Categorías Interpretación del Autor

Significados Emergentes

En su discurso A1, A2, A3, A4 y A8 se perciben que la redacción es

Currículo Estrategias importante para los autores, al de Redacción momento de desarrollar sus escritos científicos. La educación es quien potencia al hombre en esa búsqueda, refuerza el esquema $u$ orden de actuación en que ha de basarse, perfeccionarse y mejorarse a sí mismo. En la actualidad, se observa un incremento en literatura acerca del planteamiento de cómo mejorar la redacción científica, a partir de la motivación, propia experiencia de redactar, del intercambio de opiniones o experiencias con otros expertos. La sociedad del siglo XXI exige una renovación permanente de las universidades, debido a que el alcance científico y tecnológico demanda claridad, precisión, brevedad, además, cómo, cuándo y para qué escribe. Lo cual, involucra una acción lingüística que inserta el lugar social, destinatario, enunciador y finalidad. Es importante conocer los niveles de comunicación científica, aunque converjan diferencias, del mismo modo que las experiencias culturales; por esta razón, conocer las competencias comunicativas, competencia textual, estándares y políticas internacionales ayudará a la transformación del conocimiento científico significativo de calidad. No obstante, esto implica una reorganización para mejorar lo anterior, donde se aporte a su comunidad una manera diferente de crear ciencia, en cuyo centro se encuentra el proceso de apropiación del estudiante-autor. En resumen, la redacción científica es una habilidad que se alcanza y perfecciona si se asocian el dominio del idioma, enfoque, revisión, precisión, claridad, brevedad y formalidad.

Nota. Las estrategias de redacción científica ofrecen un grado de precisión y claridad del manuscrito, elaboración propia (2020).

De lo plasmado en la constitución conceptual "Estrategias de Redacción", se evidenció la existencia una serie de dificultades que enfrentan los autores al escribir un artículo científico, entre ellas: densidad informativa, complejidad sintáctica, distribución de la información (Jerz, 2000), género discursivo, estrategias (Remerger, 2000), lógica, 
incongruencias relativas a la disciplina o audiencia, plagio, debilidades gramáticas, vocabulario (Feldman, Anderson \& Mangurian, 2001) y redacción de la introducción, marco de referencia (Lerner \& Ogren-Balkama, 2007). Por ende, en la redacción se hace necesario un conocimiento previo para expresar adecuadamente la información, ver Tabla 4.

\section{Tabla 4}

Categoría: Cultura Informacional

\section{Matriz I: Análisis e Interpretación de los resultados}

\begin{tabular}{ccc}
\hline $\begin{array}{c}\text { Unidad de } \\
\text { Significados }\end{array}$ & $\begin{array}{c}\text { Categorías } \\
\text { Emergentes }\end{array}$ & Interpretación del Autor \\
\hline & & La autora interpreto lo expuesto
\end{tabular}
por A1, A2, A3, A4, A6, A8, A9 y

Cultura Habilidad A10; el deseo de obtener Informacional en la competencias, estrategias y Escritura educación continua significativa sobre cómo redactar artículos científicos. En primer lugar, se los actores desean una enseñanza en calidad de componente transformador para entender, interpretar, argumentar, proponer y generar conocimientos del área del saber correspondiente. En segundo lugar, ambicionan competencias para conocer las etapas (pre-escritura, escritura y re-escritura), producción del texto (creatividad y reelaboración), habilidades académicas (interpretación de datos, vincular la información y competencia para sintetizar) y cognitivas (planificar, releer, revisar, selección y forma). Por último, estrategias para mejorar la redacción en cuanto a estructuras gramaticales (sintácticas, semánticas, pragmáticas, estilísticas, esquemáticas y conexión mutua). Por ello, la construcción de un texto envuelve etapas para optimizar el discurso de comunicación. La práctica de lectoescritura se encuentra ligada al ámbito académico, el mismo envuelve leer, tomar apuntes y releer para producir escritos con discurso retórico fiable. En resumen, la habilidad para escribir implica un estrecho vínculo entre la organización de ideas y declaración de información, por lo que, cada letra, palabra, frase u oración se empalman para construir una coherencia temática a divulgar.

Nota. La habilidad de escrita conlleva un proceso arduo, que está determinado para las habilidades de la persona al escribir, elaboración propia (2020).

En esta estructura conceptual, unidad de significados "Cultura informacional" se consideró necesario una alfabetización científica que fortalezca las habilidades de escritura de manera significativa. Una educación basada en la construcción de documentos científicos con habilidades de razonamiento, que caracterizan el enfoque cognitivo. De acuerdo con el autor (González, 1993) "las habilidades de tipo mecánico, habilidades de tipo ortográficos, habilidades de producción, habilidades lingüísticas y habilidades organizativas" (p.39). Estas habilidades son letra legible, adecuada redacción, ortografía, ideas, componentes lingüísticos, coherencia lógica, secuencial y organizacional.

\section{Figura 1}



Nota. Integración de las ocho (4) unidades de significados que generó la red semántica de las diecinueve (12) categorías emergentes, elaboración propia (2020).

De esta forma, podemos finalizar con una ilustración Figura 1, que corresponde a la integración del cuadro (4) unidades de significados que generó la red semántica de las doce (12) categorías emergentes, correspondiente al discurso de las entrevistas realizadas sobre la importancia de generar conocimiento en el siglo XXI.

\section{Discusión}

Aun cuando, en el presente se publiquen artículos científicos muchos de ellos son rechazados por no presentar una redacción calidad, por consiguiente esto se ha convertido en un factor clave para la divulgación del conocimiento científico, que sustentado por el autor Furio et al. (2000) este es un proceso prolongado de colaboración directa entre el profesorado y los expertos en didáctica de las ciencias. Paradójicamente, la mayor parte de las investigaciones relacionadas con la producción científica son de estudiantes universitarios, con una marcada debilidad en el léxico; este planteamiento no ha sido suficientemente 
investigado en cuanto el rol que deben cumplir las instituciones, organizaciones y empresas educativas en el fortalecimiento de la redacción de los artículos científicos originales y su vínculo con el origen para proponer posibles soluciones.

Lo planteado por los autores pudiera derivarse por un lado de las cadenas burocráticas de cada editorial, al conocimiento que es más accesible o por la propagación vertiginosa del analfabetismo incitado por los grupos anacrónicos de la vieja escuela, aquellos que han creado confusión en el qué de los sucesos, para expandir su hegemonía. En vista de que la gran mayoría de los países hispanohablantes, el arte de redactar artículos científicos es considerada parte de la asignatura metodología de la Investigación, aunque no se aborde como una unidad autónoma donde se muestren las diferencias existen entre redactar un artículo y una tesis. El autor Cassany (1993) señala "no hay brebajes mágicos ni siquiera recetas instantáneas para escribir" ( $p$. 237) lo que exhibe escribir envuelve la actitud y estilo de trabajo del autor.

En los hallazgos se evidencian la interpretación, comprensión y edificación de nuevos vínculos conceptuales. De acuerdo con Moya (2003) "existen técnicas diversas para activar los conocimientos previos, como actividades de previsión, guías de anticipación, mapas semánticos, discusión, etc.” (p. 34), por este motivo se hace imprescindible alfabetizar para alcanzar la producción del conocimiento de calidad. Otro de los resultados que reclama atención en este estudio es la habilidad en escritura, donde se evidenció mayor dificultad para expresar ideas intrincadas de modo simple, demostrándose en la segunda revisión que los autores sociales lograron determinar sus propias debilidades, en cuanto a: competencias léxicas, comunicación, experiencia, preparación profesional, conocimiento significativo, estrategias de redacción, resultados de investigación, métodos, conocimiento científico, creación de contenidos, habilidad de escritura y recursos didácticos.

Resulta pertinente enfatizar que la presente investigación es oportuna y novedosa porque está orientada en la determinación de las principales causas de redacción de artículos científicos de un grupo de autores. En la actualidad, se han llevado a cabo diversas investigaciones con respecto a la relevancia en producción científica, pero estas se han presentado como un estudio general y no específico sobre las dificultades de la escritura que se oponen al incremento de la calidad. Ante las maneras de acceder a una cultura informacional, las asociaciones del sector cultural deberían reflexionar sobre cuál será su rol para instaurar una sociedad digital más culta, comprometida e interactiva (Celaya, 2011).

Una de las restricciones de esta publicación se sujeta al período de recopilación de datos, el cual se extendió desde abril hasta agosto de 2020, debido a los problemas suscitados por la pandemia mundial, de tal forma que los artículos recibidos con demora no alcanzaron a ser observados. Para la correcta interpretación de los resultados se sustenta una inclinación significativa; las dificultades encontradas se restringen por las unidades de significados a valorar en el instrumento aplicado, del mismo modo que el tiempo de revisión no fue el mismo para todos los actores sociales.

\section{Agradecimiento}

A la Dra. Rafaela Solís-Muñoz, PhD., facilitadora del Posdoctorado en Ciencia, Dra. Nora Panza de Ferrer, PhD., tutora de este valioso emprendiendo y Universidad Centro Panamericano de Estudios Superiores (UNICEPES) por esta gran iniciativa en alfabetización científica.

\section{Conclusión}

La investigación realizada permite deducir que toda propuesta de alfabetización científica plantea una declaración explícita de sus finalidades porque éstas son una condición necesaria para brindar sentido al proceso de escritura científica. Estos propósitos nacen de la teoría del currículo y de las creencias que sustente la materia. Además, se anteponen los principios, valores y normas para así dotar de estilo propio a la institución "como dinámica de un desarrollo personal que cada sujeto hace por sus propios medios, cada uno se forma a sí mismo con la ayuda de mediaciones que sólo posibilitan la formación" (Ferry, 1997, p.13). Por consiguiente, alfabetización científica (AC) no 
solo está centrada en el factor común de la actividad científica del cómo, sino también en el fortalecimiento científico a través de las Instituciones de Educación Superior, grupos, organizaciones y empresas educativas.

El propósito de educar en redacción científica resucita impostergable, es necesario apropiarse de un léxico prolijo que trascienda redaccionalmente a través de argumentos claros, auténticos y originales, que cautiven no solo a ser leídos, sino, que inciten a quienes todavía no escriben, con el fin de nutrirse de aspectos para fortalecer la redacción. Tal como lo señala el autor Foucault (1970) el "discurso científico, puede definir los axiomas que le son necesarios, los elementos que utiliza, las estructuras proposicionales que son para él legítimas y las transformaciones que acepta" (p. 314). Por ende, una educación propuesta para alfabetizar admite contribuir a una enseñanza para la ciudadanía, puesto que cualquier reforma incluye como unidad central a los docentes y estudiantes con fines científicos, un debate que casi siempre se le ha substraído excesivas veces

\section{Referencias}

Abd-El-Khalick, F., Bell, R.L. \& Lederman, N.G. (1998). The nature of science and instructional practice: Making the unnatural natural. Science Education, 82(4), 417-436.

Aguilar, T. (1999). Alfabetización científica y educación para la ciudadanía. Narcea Ediciones.

Aguirre, J., \& Jaramillo, L. (2013). Tesis de la carga teórica de la observación y constructivismo. Cinta de Moebio, 47, 7482. $554 X 2013000200002$

Alfonso-Sánchez, I (2001). El arte de escribir. Acimed, 9(2),7-85. http://www.bvs.sld.cu/revistas/aci/vol9_2_02/aci01201.h tml

Bolio, A. (2012). Husserl y la fenomenología trascendental: Perspectivas del sujeto en las ciencias del siglo XX. Reencuentro, http://www.redalyc.org/articulo.oa?id=34024824004

Caamaño, A; Gómez-Crespo, M. A.; Gutiérrez-Julián, M. S; Llopis, R. \& Martín-Díaz, M.J., (2001). El Proyecto Química Salters: un enfoque ciencia, tecnología, sociedad para la química del bachillerato, en Enseñanza de las ciencias desde la perspectiva Ciencia/Tecnologia/Sociedad. Formación científica para la ciudadanía. Narcea.

Cassany, D. (1995). La cocina de la escritura. Editorial Anagrama.
Celaya, J. (2011). Escasa creación original, colaborativa, participativa. Telos, Cuadernos de Comunicación e Innovación, $\quad n .^{\circ} 89$. https://publiadmin.fundaciontelefonica.com/index.php/p ublicaciones/add descargas?tipo fichero $=$ pdf\&idioma $\mathrm{f}$ ichero $=\&$ title $=$ TELOS $+88 \& \operatorname{code}=256 \&$ lang $=$ es $\&$ file $=\mathrm{t}$ elos_88_.pdf

Feldman, S., Anderson, V. \& Mangurian, L. (2001). Teaching effective scientific writing. Journal of College Science Teaching, 30(7), 446-450.

Ferry G. (1997). Pedagogía de la formación. Ediciones Novedades Educativas y Universidad de Buenos Aires.

Foucault, M. (1970). La arqueología del saber. Siglo XXI.

Furió, C., Vilches, A., Guisasola, J. \& Romo, V. (2001). Finalidades de la enseñanza de las ciencias en la Secundaria Obligatoria. ¿Alfabetización científica o preparación propedéutica? Enseñanza de las Ciencias, 19(3), 365-376.

Gopen, G. \& Swan, J. (1990). The Science of scientific writing.

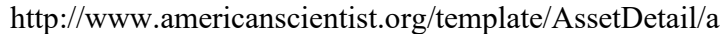
ssetid/23947?fulltext=true\&print=yes

Heidegger, M. (2006). Introducción a la fenomenología de la religión. Ediciones Siruela.

Husserl, E. (1998). Invitación a la fenomenología. Paidós.

Jerz, D. (2000). Top five problems. http://jerz.setonhill.edu/writing/technical/resume/tips.ht $\mathrm{m}$

Laugksch, R.C. (2000). Scientific Literacy: A Conceptual Overview. Science Education, 84(1), 71-94.

Lerner, N. \& Ogren-Balkama, M. (2007). Teaching scientific http://appl003.lsu.edu/acadaff/cxcweb.nsf/\$Content/Sum mer+Institute+Materials/ \$file/SI 05--Lerner-Writing_1.pdf

Martín-Díaz, M.J. (2001). Enseñanza de las ciencias ¿para qué?, Revista Electrónica de Enseñanza de las Ciencias, vol.1, n.2. 2002.

http://www.saum.uvigo.es/reec/volumenes.htm

Martínez, M. (1996). Investigación cualitativa. El comportamiento humano. ( $2^{\mathrm{a}}$ ed.). Trillas.

McConney A., Oliver M.C., Woods-McConney A., Schibeci R. \& Maor D. (2014). Inquiry, Engagement, and Literacy in Science: A Retrospective, Cross-National Analysis Using PISA 2006. Science Education 98(6), 963-980.

Moya, J. (2003). El lenguaje cientifico y la lectura comprensiva en el área de ciencias. Gobierno de Navarra Departamento de Educación y Cultura.

Olmedo-Estrada, J. C. (2011). Educación y divulgación de la Ciencia: tendiendo puentes hacia la alfabetización científica. Revista Eureka Sobre Enseñanza $Y$ Divulgación De Las Ciencias, 8(2), 137-148. https://revistas.uca.es/index.php/eureka/article/view/270 3 
Rizo-Patrón, R. (2015). Superveniencia o nacimiento trascendental. Ápeiron: Estudios de filosofía: Filosofía y
fenomenología,
(3),
381-397.

https://dialnet.unirioja.es/servlet/articulo?codigo $=59664$

62

Romberger, J. (2000). Teaching scientific writing conventions: Learning to write is an integral part of writing to learn in the Sciences. http://owl.english.purdue.edu/handouts/WAC/abstract.ht $\mathrm{ml}$

San Martín, J. (1986). La estructura del método fenomenológico. Universidad Nacional de Educación a Distancia.

Sternberg, R. \& Lubart T. (1997). La creatividad en una cultura conformista. Editorial Paidós. 